\title{
Improved DGA Methods of Power Transformer Fault Diagnosis: A Review
}

\author{
Chen-hang GE', a, Hao-yang CUI*1, b, Si-jia HUO'1, c, Wen-cheng GUO', d, \\ Hong-wei MA ${ }^{1, e}$, Lun-ming QIN ${ }^{1, f}$ \\ ${ }^{1}$ College of Electronic and Information Engineering, Shanghai University of Electric Power, \\ Shanghai 200090, China \\ agech93@163.com, bcuihy@shiep.edu.cn, c13572401905@163.com, d466862032@qq.com, \\ e1142535331@qq.com, f99917604@qq.com
}

Keywords: DGA technique, Power Transformer Fault Diagnose, Al method, statistics method, new diagnostic methods. Shanghai University of Electric Power

Abstract. Power transformers are key components in the stable operation of electric power system. Reasonable arrangements of maintenance strategy according to transformers' working condition can decrease the loss lead by transformers' faults. The dissolved gas analysis (DGA) is the widest technique used in transformers' fault diagnosis and condition monitoring. Based on the conception of DGA technique, researchers and engineers have developed many methods and standard for faults diagnosis, such as IEC standard code, IEEE ratio, and Duval triangle method. They are practical and easy to use,

but still face some problems. Many improvements for DGA have been carried out for improving the diagnostic accuracy. Artificial Intelligence (AI) method, statistics method or new diagnostic ways are the hot field for research. This paper has introduced the improved DGA methods of power transformer fault diagnosis in recent years and put forward some technical outlook of this field

\section{Introduction}

Power transformers are the vital and expensive components in electric power systems. Their proper functioning is essential for maintaining the availability and safety of electric energy supply, and preventing financial loss [1]. Long-term operation for transformers will cause internal insulation aging due to external conditions, which can lead to incipient electrical and thermal faults and result in irreparable loss. With the gradual promotion of state-based maintenance strategies in the power system industry, through the condition assessment for transformer operating conditions, timely implementation of corresponding maintenance or repair strategies can effectively reduce the losses caused by faults. For power transformers filled with mineral insulating oil (MIO), dissolved gas analysis (DGA) has been the widest technique for transformer fault diagnosis. Based on the real gas data with according faults, researchers have proposed a variety of DGA-based transformer insulation fault diagnosis standards, including the IEEE standard, Rogers ratio method, characteristic gas method, David triangle method.

The conventional DGA methods mostly achieve diagnosis through coding. Among the current commonly used methods, there are problems such as absolute code boundaries, missing codes, and susceptibility to fluctuations in gas volume fraction [2]. AI algorithms, like Clustering Based Method (CBT), Fuzzy Logic (FL), Artificial Neural Network (ANN), have been used for improve these problems. But their limitations were exposed in the study. CBT algorithms are difficult to establish the relationship between the sub-category and the fault to be diagnosed, while the distance threshold is difficult to set; the rules and membership functions in FL algorithm depends on researchers' experience. ANN algorithm uses a specific function to classify data during faults classification, which is difficult to effectively determine the data near the boundary. In addition, many parameters setting involved in the intelligent algorithm are dependent on the researchers, which severely constrains the accuracy of the diagnosis. With the development in sensors, macromolecules, and chromatography, on-line monitoring of dissolved gases in transformer oil has advanced significantly [3,4]. With the 
increasing amount of data, by mining the information in gas data which is related to the type of fault, improving the traditional method or discover new correlation characteristics according to faults principle has been a research hotspot $[5,6]$.

\section{Application of AI algorithm combining DGA technology}

With the development of computer technology and emergence of intelligent algorithms, applying AI algorithm to transformer fault diagnosis has become one of the trends in the research field. Because the dissolved gas in the oil can be obtained during the operation of the transformer, whose collection is convenient and has accumulated a large amount of data till now. The unique advantages can effectively exploit the effect of AI algorithm, which need ratios sample with high quality, large quantity and equilibrious distribution feature. So combining the DGA technique and AI algorithm is practicable and meaningful.

Researchers often "transplant" AI algorithms applied in other fields to transformer fault diagnosis, or combine two or more intelligent algorithms to overcome the disadvantages of both parties and improve the ability. Guo combines semi-supervised classifying (SSC) method, which can enforce the learning ability with a small amount of labeled data and a large amount of no-labeled data, and fuzzy nearest neighborhood label propagation (FNNLP) method as well as DGA technique for transformers faults' identification, whose accuracy can reach 91.2\% [7]. Through non-supervised or semi-supervised learning methods, deep learning (DL) is highly efficient and has achieved major research breakthroughs in various fields. Taking advantage of high efficiency for features extraction but won't be overfitting, Dai use deep belief network to diagnose, which can represent a high diagnostic accuracy [8], the typical process of DL can be represented as Fig.1 shows, where RBM is the restricted Boltzmann machine. Shakeb implemented the FL method and Adaptive Neuro fuzzy inference system (ANFIS) for circumventing limitations of conventional methods like IEC standard, who presents a reliable system with relative high accuracy [9]. Hasmat represented a gene expression programming (GEP) algorithm-based method for DGA interpretation, the accuracy with 522 DGA cases analysis shows the effectiveness [10]. Li integrated multi-classification algorithm self-adaptive evolutionary extreme learning machine (SaE-ELM) and a simple arctangent transform (AT) to improve the diagnostic accuracy on algorithm and data structure. The test analysis has shown that it has significantly improved the accuracies [11].

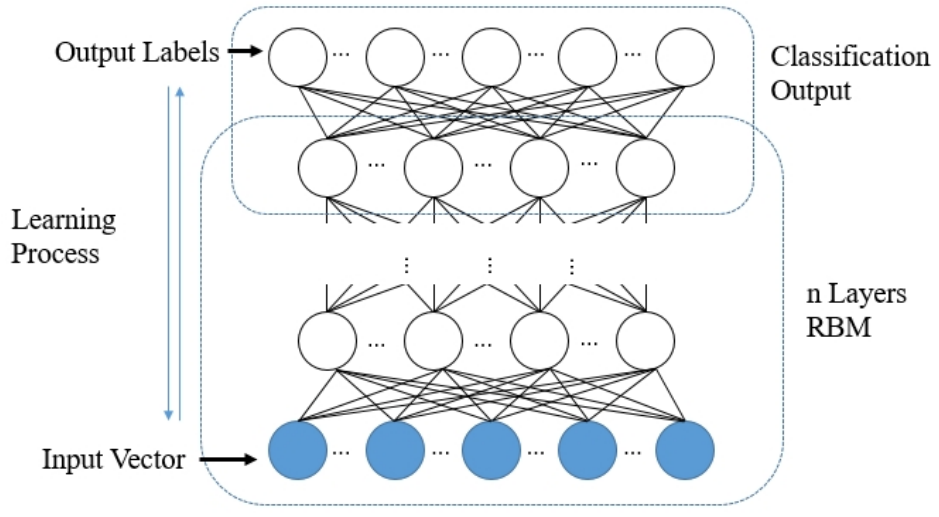

Fig.1 A typical DBN application structure

AI algorithm has been developed in assessment and diagnosis of electrical equipment for its powerful ability of fitting and generalizing. Besides, these methods can update itself with the upgrading database. However, the constraints from data quality, quantity and distribution for them have severely influenced its implementation.

\section{Improvement of conventional DGA technique}

The idea of setting specific code based on gas ratios was first proposed by Dornenberg and 
revised with Rogers before the revision of IEC standard 60599 [12]. Since then, the code diagnostic methods become the most widely used method in engineering practice, the main concept is that when the code is consistent with the fault code, the fault type can be identified. But the method will be invalid when there is no established code rule, and it is subject to gas data fluctuation and absolute code boundary.

According to IEC standard 60599, Guide to the analysis and the diagnosis of gases dissolved in transformer oil GB/T 7252-2001 has improved the old version through adding fault types and codes. But 011 code is still missing. Song applies linear regression analysis to explore the 011 code. As there is a relatively high correlation between adjacent space, by taking logarithm and measuring the linear relationship with correlation coefficient under 0.05 significance level, Song discovered a strong linear relationship among $\mathrm{H}_{2}, \mathrm{CH}_{4}, \mathrm{C}_{2} \mathrm{H}_{6}, \mathrm{C}_{2} \mathrm{H}_{4}$ [5]. Besides, Song noticed that for some fault types, there are same trend of increase or decrease for gases, but not for the other types [13]. Wang used leave-one-out (LOOM) method for selecting typical ratios by comparing the accuracy of diagnosis with different ratios group [14]. Tang employing the difficulty of feature recognition in different subspaces to complementarily filter DGA ratios for improve the accuracy of classification [15].

Improvement of traditional code methods have its own advantages in practice. However, these methods generally only valid for some specific gases data Due to the large variety of code methods, most of them miss codes or be sensitive to external conditions, there is still much room for research.

\section{Statistical methods for DGA interpretation}

The Dave triangle method is a graphic diagnosis method proposed for circumstance the drawbacks in traditional code method. With the triangle area divided by concentration percentage of $\mathrm{CH}_{4}, \mathrm{C}_{2} \mathrm{H}_{4}, \mathrm{C}_{2} \mathrm{H}_{2}$, the intersection point of the triangle fault zone is used for fault diagnosis.

Based on the fault data, Mansour designed a pentagonal visual graphics for fault diagnose. In the pentagon, all five endpoints are set by former knowledge and real fault gas data. Placing gases adjacent based on their cause and calculating the correlation coefficient for the gas type, then the pentagon can be drawn and divided. In the case verification, this method effectively solves the shortcomings of the Davul triangle method, which is difficult to diagnose low temperature overheating and partial discharge faults [16]. Besides, Gouda has drawn a heptagonal visual graphics for fault diagnose based on hydrocarbon gas and carbon oxides [17]. Moodley drawn a triangular graphics for MIO low energy degradation with $\mathrm{CH}_{4}, \mathrm{H}_{2}, \mathrm{CO}$ gases data [18].

With a variety of DGA data, a visual graphics can cover the entire fault area on the premise of containing the studied faults. The disadvantage is that it will still be affected by the difficulty of making a correct judgment when the data is located at the boundary of each fault.

\section{Conclusion and prospect}

Among the diagnostic methods mentioned above, code methods and visual graphics methods are mainly constructed based on empirical data, while the AI methods achieve the diagnosis by mining the correlation between gas data and fault types. Compared with the code methods, visual graphics methods are relatively better but still have a great room for improvement. The main problems for these two way are absolute boundary and susceptibility to gas volume fraction, which need to be solved fundamentally. AI methods can have a positive influence for improving this, but how to select appropriate functions, determine parameters, and utilize the data is its biggest difficulties. In addition, with the increasing data collecting ways and the decreasing complexity, the parameters related to the condition of the transformer are also surpassing the DGA data, a reasonable way to use these new monitoring data can have a significant improvement on effective diagnosis. 


\section{Acknowledgements}

This work was financially supported by the National Natural Science Foundation of China (61107081), Local Colleges and Universities Capacity Building Program (15110500900).

\section{References}

[1] M. Duval. Dissolved Gas Analysis: It Can Save Your Transformer. IEEE Electr. Insul. Mag. Vol. 5 (1989), p. 51-59

[2] Guide to the Interpretation of Dissolved and Free Gases Analysis, IEC Publ, 2007.

[3] Yang Jian-hua, Hou Hong, Wang Lei and Liu Fu. Electronic nose system based on integrated gas sensors array. Journal of Transducer Technology. Vol. 22 (2003) p. 21-23

[4] Zhang Chuan, Wang Fu. Application of Photo-Acoustic Spectroscopy Technology to Dissolved Gas Analysis in Oil of Oil-Immersed Power Transformer. High Voltage Engineering. Vol. 31 (2005) p. $84-86$

[5] Song Bin, Liu Zhixiong, Li Enwen, Wang Guoli. Preliminary study of undefined three-ratio code in DGA. Electric Power Automation Equipment. Vol. 35 (2015), p. 60-65

[6] Vinicius G. M. Cruz, Andre L. H. Costa, Marcio L. L. Paredes. Development and evaluation of a new DGA diagnostic method based on thermodynamics fundamentals. IEEE Transactions on Dielectrics and Electrical Insulation. Vol. 22 (2015) p. 888-894

[7] Guo Xincheng, Song Qiong, Fan Xiuling. Transformer Fault Diagnosis Based on Semi-supervised Classifying Method. High Voltage Engineering. Vol. 39 (2013), p. 1096-1100

[8] J. Dai, H. Song, G. Sheng, X. Jiang. Dissolved gas analysis of insulating oil for power transformer fault diagnosis with deep belief network. IEEE Transactions on Dielectrics and Electrical Insulation. Vol. 24 (2017) p.2828-2835

[9] S. A. Khan, M. D. Equbal and T. Islam. A comprehensive comparative study of DGA based transformer fault diagnosis using fuzzy logic and ANFIS models. IEEE Transactions on Dielectrics and Electrical Insulation. Vol. 22 (2015) p. 590-596

[10]H. Malik and S. Mishra. Application of Gene Expression Programming (GEP) in Power Transformers Fault Diagnosis Using DGA. IEEE Transactions on Industry Applications. Vol. 52 (2016) p. 4556-4565

[11] S. Li, G. Wu, B. Gao, C. Hao, D. Xin and X. Yin. Interpretation of DGA for transformer fault diagnosis with complementary SaE-ELM and arctangent transform. IEEE Transactions on Dielectrics and Electrical Insulation. Vol. 23 (2016) p. 586-595

[12] Mineral Oil-Impregnated Electrical Equipment in Service - Guide to the Interpretation of Dissolved and Free Gases Analysis. IEC Standard. 1999.

[13] Song Bin, Liu Zhixiong, Li Enwen and Wang Guoli. Dissolved Gas Analysis Based on Negative Relational Grade. Power System Technology. Vol. 39 (2015) p.2627-2632

[14] Wang Ke, Li Jinzhong, Zhang Shuqi, Sun Jiantao, Wang Jianyi, Gao Fei and Cheng Huanchao. New Features Derived from Dissolved Gas Analysis for Fault Diagnosis of Power Transformers. Proceedings of the CSEE. Vol. 36 (2016) p. 6570-6578+6625

[15] Tang Yongbo, Gui Weihua, Peng Tao and Ou Angwei. Transformer Fault Diagnosis Model Based on PCA and KICA Feature Extraction. High Voltage Engineering. Vol. 402014 p.557-563 [16] D. E. A. Mansour. Development of a new graphical technique for dissolved gas analysis in power transformers based on the five combustible gases. IEEE Transactions on Dielectrics and Electrical Insulation. Vol. 22 (2015) p. 2507-2512

[17] O. E. Gouda, S. H. El-Hoshy and H. H. El-Tamaly. Proposed heptagon graph for DGA interpretation of oil transformers. IET Generation, Transmission \& Distribution. Vol. 12 (2018) p. 490-498

[18] N. Moodley and C. T. Gaunt. Low Energy Degradation Triangle for power transformer health assessment. IEEE Transactions on Dielectrics and Electrical Insulation. Vol.24 (2017) p.639-646 